\title{
Additiver Kettenindex für die Preisbereinigung der Volkswirtschaftlichen Gesamtrechnung: Kritische Überlegungen aus aktuellem Anlass
}

\author{
Utz-Peter Reich
}

Fachhochschule Mainz

\section{Problem}

Den Nutzern der Volkswirtschaftlichen Gesamtrechnung steht eine Neuerung ins Haus. Die amtliche Statistik wird auf Anordnung der Europäischen Union die Methode, nach der sie bisher die Wachstumsraten des Bruttoinlandsprodukts einer Volkswirtschaft und damit zusammenhängender Aggregate der VGR berechnet hat, demnächst ändern. Internationalem Brauch folgend werden die zur Aggregation verwendeten Preise und Gewichte nicht mehr aus einem früheren Basisjahr, sondern zeitnah am aktuellen Rand erhoben und eingebaut. Der erfreuliche Vorteil der Aktualität wird allerdings durch einen schwerwiegenden Nachteil erkauft. Die nach der neuen Methode erstellten Aggregate sind nur noch eingeschränkt, nämlich in Jahrespaaren addierbar. Über mehrere Jahre hinweg ist die Summe der deflationierten Subaggregate (Volumina) nicht gleich dem deflationierten Gesamtaggregat. Treibt man also den Teufel eines veralteten Basisjahres aus mit dem Beelzebub mangelnder Konsistenz?

Addierbarkeit der Einzelposten ist bisher eine selbstverständliche und unverzichtbare Eigenschaft jedes wirtschaftlichen Rechenwerks gewesen. Aussagefähige Salden von Konten können nur gebildet werden, wenn die darin eingehenden Transaktionen tatsächlich alle zu einer gemeinsamen Summe zusammengezählt werden dürfen. Diese Eigenschaft nunmehr aufzugeben, wird bisher nicht ausgesprochene Folgen haben sowohl für die Kontrollierbarkeit des Rechenwerks bei seiner Erstellung als auch für die Transparenz bei der Nutzung. Die herrschende Indextheorie, die den bevorstehenden methodischen Wechsel vorbereitet hat, sieht den Nachteil mangelnder Additivität als unvermeidlich an und nimmt ihn in Kauf zugunsten der höher gewerteten Aktualität. Eine solche Prioritätensetzung mag für die Preisstatistik, dem Hauptgegenstand der Indextheorie, zutreffen, für die VGR als einem Kontensystem, das aus Additionen besteht, ist sie schwer zu handhaben. Es lohnt sich deshalb zu prüfen, ob Aktualität und Additivität sich wirklich in der bisher angenommenen, mathematisch unvermeidlichen Gegensätzlichkeit einander ausschließen, oder ob es möglicherweise einen dritten Weg gibt, der beide Eigenschaften vereinbart. Im folgenden wird gezeigt, wie durch eine leichte und gut zu interpretierende Änderung der Standardmethode von Verkettung die Aktualität der in die Rechnung eingehenden Daten hergestellt und dennoch die kontenmäßige Additivität der erhaltenen Aggregate beibehalten werden könnte.

Die hier in Frage stehenden methodischen Ansätze („Formeln“) können verbal kurz wie folgt charakterisiert werden: 
Laspeyres-Index: Das Volumen der Berichtsperiode wird mittels Division des Nominalwerts durch den zugehörigen Paasche-Preisindex (bezogen auf die Basisperiode) errechnet.

Kettenindex, traditionell: Das Volumen der Berichtsperiode wird zunächst jeweils zu Preisen der Vorperiode errechnet, und diese werden durch Verkettung in die Preise der Basisperiode überführt.

Kettenindex, Vorschlag: Das Volumen der Berichtsperiode wird zu Preisen der Vorperiode errechnet, und diese werden durch eine monetäre Korrektur, die die Variabilität der verwendeten Recheneinheit berücksichtigt, auf die Basisperiode zurückgeführt.

\section{Analyse}

Ausgangspunkt sei der oft gebrauchte Ausdruck für das Volumen ,in konstanten Preisen“.

Dieser Laspeyres-Index eines Volumenaggregates eines Nominalwerts $C^{t}$ zum Zeitpunkt $t$ ist definiert als

$$
\mathrm{Q}_{\mathrm{C}}{ }^{\mathrm{t}}=\sum_{\mathrm{i}=1}^{\mathrm{n}} \mathrm{p}_{\mathrm{i}}^{0} \mathrm{q}_{\mathrm{i}}^{\mathrm{t}} \text {. }
$$

Er wird üblicherweise berechnet, indem man den zugehörigen Paasche-Preisindex $\mathrm{P}_{\mathrm{C}}{ }^{\mathrm{t}}$ auf den Nominalwert $\mathrm{C}^{\mathrm{t}}$ anwendet, nämlich

$$
\begin{gathered}
P_{\mathrm{C}}^{t}=\frac{\sum_{i=1}^{n} p_{i}{ }^{t} q_{i}{ }^{t}}{\sum_{i=1}^{n} p_{i}{ }^{0} q_{i}{ }^{t}}, \\
\mathrm{Q}_{\mathrm{C}}{ }^{\mathrm{t}}=\frac{\mathrm{C}^{\mathrm{t}}}{\mathrm{P}_{\mathrm{C}}^{\mathrm{t}}}=\sum_{\mathrm{i}=1}^{\mathrm{n}} \mathrm{p}_{\mathrm{i}}^{\mathrm{t}} \mathrm{q}_{\mathrm{i}}^{\mathrm{t}}: \mathrm{P}_{\mathrm{C}}^{\mathrm{t}}=\sum_{\mathrm{i}=1}^{\mathrm{n}} \mathrm{p}_{\mathrm{i}}^{0} \mathrm{q}_{\mathrm{i}}^{\mathrm{t}} .
\end{gathered}
$$

Nominalwert geteilt durch Paasche-Preisindex ergibt das Volumen gemäß Laspeyres. Da die Formeln analog auch für ein anderes Aggregat I gelten, gilt in der Zusammensetzung

$$
\mathrm{Q}_{\mathrm{C}}^{\mathrm{t}}+\mathrm{Q}_{\mathrm{I}}^{\mathrm{t}}=\frac{\mathrm{C}^{\mathrm{t}}}{\mathrm{P}_{\mathrm{C}}^{\mathrm{t}}}+\frac{\mathrm{I}^{\mathrm{t}}}{\mathrm{P}_{\mathrm{I}}^{\mathrm{t}}}=\sum_{\mathrm{i}=1}^{\mathrm{n}} \mathrm{p}_{\mathrm{i}}^{0} \mathrm{q}_{\mathrm{i}}^{\mathrm{t}}+\sum_{\mathrm{i}=\mathrm{n}+1}^{\mathrm{n}+\mathrm{m}} \mathrm{p}_{\mathrm{i}}^{0} \mathrm{q}_{\mathrm{i}}^{\mathrm{t}}=\sum_{\mathrm{i}=1}^{\mathrm{n}+\mathrm{m}} \mathrm{p}_{\mathrm{i}}^{0} \mathrm{q}_{\mathrm{i}}^{\mathrm{t}}=\frac{\mathrm{BIP}^{\mathrm{t}}}{\mathrm{P}_{\mathrm{BIP}}^{\mathrm{t}}}=\mathrm{Q}_{\mathrm{BIP}}^{\mathrm{t}} .
$$

Die Summe der preisbereinigten Aggregate C und I ist gleich der preisbereinigten Summe BIP der nominalen Aggregate. Dies gilt, weil die Güter des Aggregates C $(\mathrm{i}=1, \ldots, \mathrm{n})$ und die des Aggregats $\mathrm{I}(\mathrm{i}=\mathrm{n}+1, \ldots, \mathrm{n}+\mathrm{m})$ mit einem von der Zeit unabhängigen Preisvektor $\mathrm{p}_{\mathrm{i}}{ }^{0}$ gewichtet werden. Gleichung 6 verliert ihre Gültigkeit, wenn man die dort benutzten Paasche-Preisindizes durch Ketten-Preisindizes ersetzt. In Formeln drückt sich das wie folgt aus.

$$
\mathrm{K}^{\mathrm{t}}=\frac{\sum \mathrm{p}^{1} \mathrm{q}^{1}}{\sum \mathrm{p}^{0} \mathrm{q}^{1}} \times \frac{\sum \mathrm{p}^{2} \mathrm{q}^{2}}{\sum \mathrm{p}^{1} \mathrm{q}^{2}} \times \ldots \times \frac{\sum \mathrm{p}^{\mathrm{t}} \mathrm{q}^{\mathrm{t}}}{\sum \mathrm{p}^{\mathrm{t}-1} \mathrm{q}^{\mathrm{t}}}
$$


ist der verkettete Preisindex, dessen einzelne Glieder jeweils Paasche-Form bezüglich des Vorjahres haben. Daraus erhält man für das verkettete Volumen $\mathrm{R}^{\mathrm{t}}$ die Gleichung

$$
\mathrm{R}_{\mathrm{C}}{ }^{\mathrm{t}}=\frac{\mathrm{C}^{\mathrm{t}}}{\mathrm{K}_{\mathrm{C}}^{\mathrm{t}}}=\sum \mathrm{p}^{0} \mathrm{q}^{0} \times \frac{\sum \mathrm{p}^{0} \mathrm{q}^{1}}{\sum \mathrm{p}^{0} \mathrm{q}^{0}} \times \ldots \times \frac{\sum \mathrm{p}^{\mathrm{t}-1} \mathrm{q}^{\mathrm{t}}}{\sum \mathrm{p}^{\mathrm{t}-1} \mathrm{q}^{\mathrm{t}-1}} .
$$

Wenn man hierzu analog das Volumen für I bildet und unabhängig für BIP, so ergibt sich

$$
\mathrm{R}_{\mathrm{C}}^{\mathrm{t}}+\mathrm{R}_{\mathrm{I}}^{\mathrm{t}}=\frac{\mathrm{C}^{\mathrm{t}}}{\mathrm{K}_{\mathrm{C}}^{\mathrm{t}}}+\frac{\mathrm{I}^{\mathrm{t}}}{\mathrm{K}_{\mathrm{I}}^{\mathrm{t}}} \neq \frac{\mathrm{BIP}^{\mathrm{t}}}{\mathrm{K}_{\mathrm{BIP}}^{\mathrm{t}}}=\mathrm{R}_{\mathrm{BIP}}^{\mathrm{t}}
$$

Diese Preisbereinigung ist nicht additiv.

Das SNA 1968 (Chapter IV - The System as a basis for quantity and price comparisons unterscheidet nun aber zwei grundsätzlich unterschiedliche Ansatze:

a) The decomposition of a change in value expressed in current prices into a price component and a quantity component.

b) Considering the purchasing power.

Sie unterscheiden sich in der Technik der Preisbereinigung, vor allem aber in der Intention:

„These two approaches are radically different and should not be confused with one another" (SNA 1968 Abschnitt 4.3). Das SNA 93 hat diese Konzeption übernommen (mit der Ausnahme, dass es sich um „Volumen“ und nicht „Mengen“ handelt). Wenn man auf dieser Basis die Konzepte der Preisstatistik analysiert, erkennt man, das dort Kaufkraftmessung auf der einen und Preismessung auf der anderen Seite in der Tat nicht getrennt, sondern in einem Index vermischt werden.

Unter dem Aspekt der Trennung von Preisänderung und Kaufkraftänderung ist der direkt aus der Preisstatistik übernommene Preisindex $p_{i}(t)$ kritisch zu sehen. Dieser absolute oder nominale Index kann sich nämlich aus zwei ganz unterschiedlichen Gründen ändern, es kann der echte Preis im Sinne der Preistheorie sein, der das Gut $\mathrm{i}$ relativ zu anderen Gütern teurer oder billiger macht, oder es kann die Geldentwertung sein, die alle Güter zugleich trifft und damit den Maßstab ändert, in dem Preise überhaupt gemessen werden. Der nominale Preis als „Euro/Stück“ ist daher zu zwei unterschiedlichen Jahren nicht vergleichbar, die entsprechenden Transaktionen sind nominal nicht addierbar. Vergleichbar sind die Preise verschiedener Jahre erst, wenn sie um die Maßstabsänderung der Recheneinheit, in der sie gemessen werden, korrigiert sind, also als „Realpreise“, in der Terminologie des SNA. Sie seien definiert als

$$
\pi_{i}^{t}=\frac{p_{i}^{t}}{P^{t}}
$$

wobei $\mathrm{P}^{\mathrm{t}}$ die Maßstabsänderung der Geldeinheit beschreibt, die üblicherweise als Kaufkraftänderung gegenüber einem definierten Warenkorb (privater Konsum oder BIP) bestimmt wird. 


\section{Operationalisierung}

Um das dargelegte Konzept der Trennung von relativem Preis des Gutes und Kaufkraft der Währung in der VGR umzusetzen, muss man Gleichung 6, in der das verkettete Volumen definiert wird, noch einmal betrachten. Wie sie da steht, ist sie wie folgt zu interpretieren: Man geht vom Nominalwert des Basisjahres aus und multipliziert diesen mit dem Wachstumsfaktor jedes Jahres, der sich ergibt, wenn man die Volumenänderungen jeweils zu Preisen des Vorjahres misst. Man kann nun dieselbe Gleichung auch anders interpretieren, indem man sie etwas umformt. Wenn man mit

$$
\mathrm{P}^{01}=\frac{\sum \mathrm{p}^{1} \mathrm{q}^{1}}{\sum \mathrm{p}^{0} \mathrm{q}^{1}}
$$

den Paasche-Preisindex zwischen Jahr 0 und Jahr 1 bezeichnet, kann man Gleichung 6 auch als

$$
\mathrm{R}_{\mathrm{C}}^{\mathrm{t}}=\frac{1}{\mathrm{P}_{\mathrm{C}}{ }^{01} \times \mathrm{P}_{\mathrm{C}}{ }^{12} \times \ldots \times \mathrm{P}_{\mathrm{C}}^{\mathrm{t}-2, \mathrm{t}-1}} \sum_{\mathrm{i}=1}^{\mathrm{n}} \mathrm{p}^{\mathrm{t}-1} \mathrm{q}^{\mathrm{t}}
$$

schreiben. Dabei ist der zweite Term auf der rechten Seite als Volumen in Preisen des Vorjahres zu lesen und der linke Term als Rückführung dieser Preise auf die Basis des Jahres 0. Analog kann man für das Aggregat der Investitionen schreiben

$$
\mathrm{R}_{\mathrm{I}}^{\mathrm{t}}=\frac{1}{\mathrm{P}_{\mathrm{I}}^{01} \times \mathrm{P}_{\mathrm{I}}^{12} \times \ldots \times \mathrm{P}_{\mathrm{I}}^{\mathrm{t}-2, \mathrm{t}-1}} \sum_{\mathrm{i}=\mathrm{n}+1}^{\mathrm{n}+\mathrm{m}} \mathrm{p}^{\mathrm{t}-1} \mathrm{q}^{\mathrm{t}}
$$

Man erkennt hier den Grund der Ungleichung 7. Bezüglich des rechten Terms wäre die Summe aus Gleichungen 10 und 11 additiv, aber der jeweils vorgeschaltete unterschiedliche Preisindex verhindert das. Das legt den Gedanken nahe, statt der vielen verschiedenen einen gemeinsamen einheitlichen Preisindex anzuwenden, um die Nominalpreise verschiedener Perioden in Realpreise $\mathrm{zu}$ überführen und damit vergleichbar zu machen. Gebräuchliche Indikatoren sind der Verbraucher-Preisindex $\mathrm{P}_{\mathrm{C}}$ oder der BIP-Deflator $\mathrm{P}_{\mathrm{BIP}}$. Für die VGR bietet sich der letztere an, weil dann Inflations- und Wachstumsmessung integriert sind.

Damit kommen wir zu dem Vorschlag, die übliche Verkettung gemäß Gleichungen 12 und 13 zu ersetzen durch

$$
\mathrm{R}_{\mathrm{C}}^{\mathrm{t}}=\frac{1}{\mathrm{P}_{\mathrm{BIP}}{ }^{01} \times \mathrm{P}_{\mathrm{BIP}}{ }^{12} \times \ldots \times \mathrm{P}_{\mathrm{BIP}}{ }^{\mathrm{t}-2, \mathrm{t}-1}} \sum_{\mathrm{i}=1}^{\mathrm{n}} \mathrm{p}^{\mathrm{t}-1} \mathrm{q}^{\mathrm{t}}
$$

und

$$
\mathrm{R}_{\mathrm{I}}^{\mathrm{t}}=\frac{1}{\mathrm{P}_{\mathrm{BIP}}{ }^{01} \times \mathrm{P}_{\text {BIP }}{ }^{12} \times \ldots \times \mathrm{P}_{\mathrm{BIP}}{ }^{\mathrm{t}-2, \mathrm{t}-1}} \sum_{\mathrm{i}=\mathrm{n}+1}^{\mathrm{n}+\mathrm{m}} \mathrm{p}^{\mathrm{t}-1} \mathrm{q}^{\mathrm{t}} .
$$

Gleichungen 12 und 13 sind addierbar. 


\section{Kritik}

Es ist nicht anzunehmen, dass die Väter der Kettenindexneuerung nicht wussten, welche Unbill sie dem Nutzer zumuten. Ob der Trade off zwischen dem Gewinn an Aktualität und dem Verlust an Konsistenz zugunsten ersterer ausgeht, stellt sich letzten Ende als eine politische Frage heraus, vor allem wenn man bedenkt, dass keine Alternative ohne Nachteile ist, auch nicht die hier vom Autor ventilierte. Wenn die offiziellen Erzeuger der VGR ihr folgen wollten, wäre es ein Vorteil, dass damit nicht sehr viel zusätzlicher Rechenaufwand verbunden wäre. Es müsste allerdings auch ein Wort über die gegebenen Klassifikationsstandards und ihre Bedeutung für das gegenständliche Problem erfolgen.

Es sei gewarnt, dass die hier vorstellte Methode nicht mit der reinen Kaufkraftbereinigung, wie sie üblicherweise verstanden wird, zu verwechseln ist. Es handelt sich um eine genuine Preisbereinigung, wo die Volumina $q^{t}$ mit den Preisen des Vorjahres gewichtet und addiert werden, wie beim gewöhnlichen Kettenindex. Der Unterschied besteht im Bezug dieser Preise des Vorjahres auf das Basisjahr $\mathrm{t}^{0}$. Der bisher verwendete Kettenindex benutzt dazu die Preisentwicklung des jeweiligen Aggregats, als Alternative kann man den allgemeinen Preisindex nehmen. Rechtfertigung für das letztere ist das in der Preisstatistik nicht unmittelbar gegebene und verwirklichte Gebot der Trennung von Güterpreis im eigentlichen Sinn - als Relativpreis - und allgemeiner Preisniveauänderung als monetärem Phänomen.

\section{Literatur}

SNA. System of national accounts, rev. 3, United Nations, New York, (1968).

SNA. System of national accounts, rev. 4, United Nations, New York, (1993).

Adresse des Autors:

Prof. Dr. Utz-Peter Reich

Fachhochschule Mainz

Postfach 1967

D-55009 Mainz

Germany

E-mail: utz.reich@wiwi.fh-mainz.de 